\title{
Value associations of irrelevant stimuli modify rapid visual orienting
}

\author{
Helena J. V. Rutherford, Jennifer L. O'Brien, and Jane E. Raymond \\ Bangor University, Bangor, Wales
}

\begin{abstract}
In familiar environments, goal-directed visual behavior is often performed in the presence of objects with strong, but task-irrelevant, reward or punishment associations that are acquired through prior, unrelated experience. In a two-phase experiment, we asked whether such stimuli could affect speeded visual orienting in a classic visual orienting paradigm. First, participants learned to associate faces with monetary gains, losses, or no outcomes. These faces then served as brief, peripheral, uninformative cues in an explicitly unrewarded, unpunished, speeded, target localization task. Cues preceded targets by either 100 or 1,500 msec and appeared at either the same or a different location. Regardless of interval, reward-associated cues slowed responding at cued locations, as compared with equally familiar punishment-associated or no-value cues, and had no effect when targets were presented at uncued locations. This localized effect of reward-associated cues is consistent with adaptive models of inhibition of return and suggests rapid, low-level effects of motivation on visual processing.
\end{abstract}

Most of the time, we perform visual tasks in familiar environments that are replete with motivationally salient, but currently irrelevant, objects. Motivationally salient objects are items for which we have had prior rewarding or punishing experiences. As a result, we are potentially able to predict the value of interacting with them. The question we addressed in the present study was whether irrelevant, but motivationally salient, objects can influence rapid, reflexive visual orienting. Recent neurobiological research has shown that instrumental learning, via a network involving the orbitofrontal cortex, amygdala, and ventral striatum (among other areas), leads to the acquisition of neural codes that are associated with specific stimuli and that are able to predict the outcome value of interacting with them (O'Doherty, 2004). Value prediction codes are thought to forecast outcome magnitude, probability of occurrence, and valence (rewarding vs. punishing) and thus represent the motivational value of stimuli. Importantly, they may rapidly modulate early sensory processes (Kiss, Driver, \& Eimer, 2009; Stolarova, Keil, \& Moratti, 2006) and thereby influence reflexive visual orienting. Although previous work has shown visual orienting to be enhanced by positive incentives that are associated with target stimuli (Engelmann \& Pessoa, 2007; Kiss et al., 2009; Small et al., 2005), it remains unclear whether value associations can modulate orienting when they and their associated stimuli are irrelevant. It is also unclear whether punishment is as effective as reward in this context. Reward versus punishment learning involves different neural mechanisms (Kahn et al., 2002) and has been found to produce differential benefits when learned stimuli must be selected in an attentional blink task (Raymond \& O'Brien, 2009).

Two areas offer insight into the role of value prediction on orienting: dot-probe studies in drug users and reflexive orienting studies using emotional stimuli. In the former, two images (one motivationally salient and the other neutral) are presented simultaneously. At their offset, a target (dot probe) is presented at the prior location of either the salient or nonsalient image. A faster response time (RT) to probes replacing salient versus neutral images is thought to reflect a voluntary bias of a spatially limited attentional mechanism. Biases have been found for aversively conditioned stimuli (Armony \& Dolan, 2002) and for drugrelated (rewarding) stimuli in substance-using samples (Bruce \& Jones, 2006), showing that voluntary attention can be drawn to irrelevant stimuli with positive or negative motivational value (see, e.g., Robinson \& Berridge, 1993). However, such effects may not predict effects on reflexive orienting because attentional biases are not consistently observed when images are only briefly presented (Bradley, Field, Healy, \& Mogg, 2008).

Reflexive visual orienting is generally studied using a spatial orienting task (Posner \& Cohen, 1984) in which an irrelevant (nonpredictive) peripheral cue is briefly presented prior to the presentation of a target. The target is presented at either the same location as the cue (cued target) or at a different location (uncued target). Numerous studies have established that the target RT is determined by an interaction between the location of the target (cued vs. uncued) and the interval between the onset of the cue and target (cue-target onset asynchrony, CTOA; Posner,

J. E. Raymond, j.raymond@bangor.ac.uk 
Rafal, Choate, \& Vaughan, 1985). With short CTOAs $(<300 \mathrm{msec})$, RTs are faster for targets presented at cued versus uncued locations - an effect known as facilitation. In contrast, with long CTOAs ( $>300 \mathrm{msec})$, RTs are slower for cued versus uncued targets - an effect called inhibition of return (IOR). Accounts for these effects are varied and range from spatially limited central attention mechanisms (see, e.g., Posner et al., 1985) to locally acting adaptive mechanisms (Dukewich, 2009; Sereno, Lehky, Patel, \& Peng, 2010). Of interest in the present article are studies that used nonpredictive, peripheral, emotional images as cues. Such studies (all using long CTOAs) failed to find any effect of emotional cue content (Lange, Heuer, Reinecke, Becker, \& Rinck, 2008; Stoyanova, Pratt, \& Anderson, 2007). Studies reporting emotional cue effects (e.g., Fox, Russo, \& Dutton, 2002) used predictive cues (thereby altering cue value, irrespective of emotional content) and primarily reported effects for participants scoring high in trait anxiety. Perhaps previous failures to find effects of emotional cues on reflexive orienting occurred because these nonpredictive, irrelevant cues, although nominally emotional, may have had little or no actual motivational salience for participants.

To more effectively manipulate motivational salience, we conducted a two-phase experiment that began with a simple instrumental conditioning procedure to imbue stimuli with monetary gain (reward) or loss (punishment) associations. These stimuli were then used as brief, peripheral, nonpredictive cues in a spatial orienting task requiring speeded, manual localization responses (without explicit rewards or punishments). A short (100-msec) and a long $(1,500$-msec) CTOA were used in a mixedblock design. In this task, all of the cues were equally uninformative, all had been exposed an equal number of times in the preceding learning task, and their learned value was equally irrelevant. Any differential effect of the cue's learned value would indicate that visual orienting mechanisms are influenced by value prediction codes that are associated with irrelevant stimuli. A modulation of RT to both cued and uncued targets (in opposite directions) by cue value is predicted if the RT is governed by a spatially limited attention mechanism. However, if cues act to control orienting via local adaptive processes, then the modulation of cued but not of uncued target RT is predicted. Finally, we predicted an effect of reward- but not of punishment-associated cues, on the basis of previous work showing selective sensitivity to reward- (not punishment-) associated stimuli when voluntary attention is diminished (Raymond \& O’Brien, 2009).

\section{METHOD}

\section{Participants}

Forty experimentally naive adults ( 21 females; mean age $=$ 21 years) who were recruited from Bangor University participated in exchange for course credit and money. All reported normal or corrected-to-normal visual acuity and gave informed consent.

\section{Apparatus}

A Pentium-IV computer ran E-Prime 1.1 software (Schneider, Eschman, \& Zuccolotto, 2002) to control images on a 51-cm color monitor $(85 \mathrm{~Hz}, 1,280 \times 1,024$ pixel resolution) that was viewed from approximately $60 \mathrm{~cm}$.

\section{Stimuli}

Twelve grayscale, computer-generated (GenHead 1.2; Genemation Limited, 2002-2004, Manchester, U.K.), nonexpressive, male faces $\left(3.4^{\circ} \times 3.2^{\circ}\right)$ without hair, neck, or teeth visible were used in the learning task (as were used in Raymond \& O'Brien, 2009) and as cues in the orienting task, with a black, open circle serving as the target $\left(2.6^{\circ} \times 2.6^{\circ}\right)$. Alphanumeric stimuli appeared in black 18 -point Courier New font. All of the stimuli were presented on a uniform white field.

\section{Design and Procedure}

Value learning task. Value learning involved a single block of 600 trials in which six different pairs of faces were presented 100 times each in random order. Figure 1A depicts a typical trial. Participants were instructed to maximize winnings by choosing one face (indicating the left or the right using the " $\mathrm{z}$ " or the " $\mathrm{m}$ " key, respectively). Immediately after response, feedback was provided. Two face pairs produced only gains; two produced only losses; and two never produced any outcome (serving as controls for familiarity). The running total was incremented (gains) or decremented (losses) by a constant value ( 5 pence; $\pm v$ ) with a probability $(p)$ of $.8, .2$, or 0 , depending on the face chosen (no outcome was default). Faces with .8 probability of outcome were always paired with a .2 probable-outcome face of the same valence (gain/loss). This yielded two faces each for four nominal expected values $(\mathrm{EV}$, where $\mathrm{EV}=$ $v \times p)$ and four faces with no-outcome associations. Thus, stimuli can be referred to by their nominal EVs: $-.8 v,-.2 v, 0, .2 v$, and $.8 v$, where $\mathrm{EV}$ - designates punishment association, $\mathrm{EV}+$ designates reward association, and EV0 designates a no-outcome association. Each face always appeared with the same mate, but its location across trials was random. The assignment of face exemplars to pair types and probabilities was counterbalanced across participants to prevent image effects.

Spatial orienting. Participants next engaged in the spatial orienting task (Figure 1B). A single face cue appeared for $85 \mathrm{msec}$ and then, with a CTOA of 100 or $1,500 \mathrm{msec}$, the target appeared either just below the location of the previous cue or at the corresponding location in the opposite hemifield (spatial separation was used to reduce masking effects in the short CTOA condition). Participants reported target location (using their index fingers indicating the left or the right, using the " $\mathrm{z}$ " or the " $\mathrm{m}$ " key, respectively) as quickly and accurately as possible.

Each combination of CTOA (100 msec, $1,500 \mathrm{msec})$, cue location (left, right), target location (left, right), and cue exemplar (i.e., each face used in the value learning task) was presented equally often in a random order within a single block of 480 trials (preceded by 8 practice trials).

\section{Data Analysis}

Learning. Learning was defined as choosing the optimal stimulus ( $.8 v$ for gain pairs or $-.2 v$ for loss pairs) on $65 \%$ or more of the last 30 trials of the learning phase. If this criterion was met for both gain or both loss pairs, then the RTs obtained in the orienting task using different exemplars but the same nominal EV were averaged. If only one pair was learned to criteria, then only the RTs pertaining to the successfully learned pair were used.

Spatial orienting. RTs were excluded from the analysis if the response (1) was incorrect, (2) was from the first three trials following an incorrect response, (3) occurred too quickly ( $<200 \mathrm{msec}$ ), or (4) was excessively slow $(>1,200 \mathrm{msec})$. This accounted for $<2 \%$ of the data. Response accuracy averaged $98 \%$ correct $(S D=$ $1.5 \%$ ). The RT data were further trimmed by calculating individual participant means (for each condition), then by removing outliers greater than $3 S D$ s above this value. Resultant mean RTs were used in ANOVAs only if the participant demonstrated learning for those faces. Repeated measures ANOVAs using CTOA, cue value proper- 
A

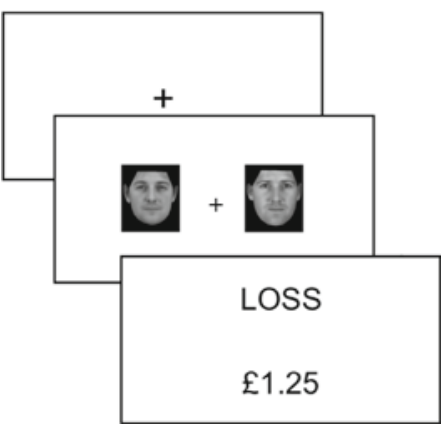

B

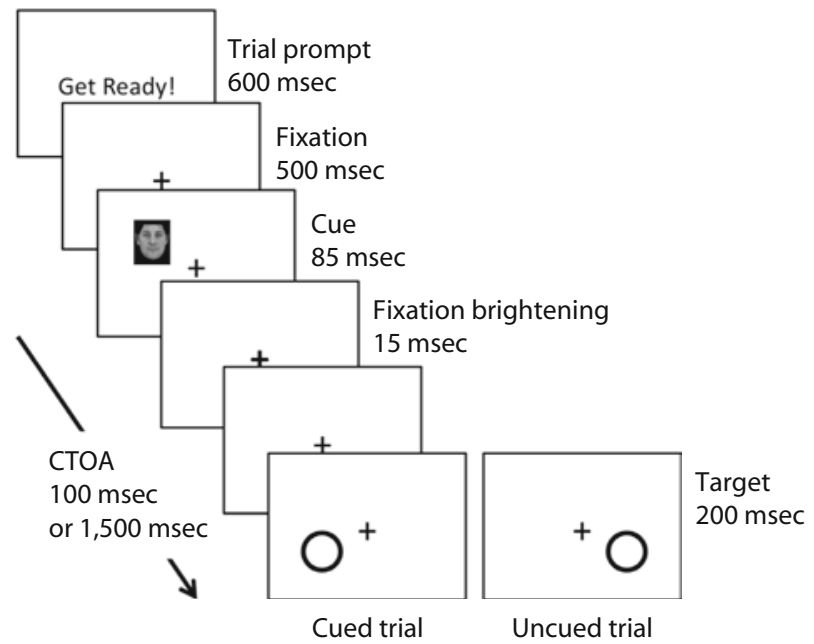

Figure 1. (A) An example trial in the value-learning task. Face pairs (presented $3.4^{\circ}$ to the left and the right of fixation) were presented and remained visible until a choice (left/right) was made. Feedback could display WIN (in green, plus a "bing" sound), LOSS (in red, plus a "bong" sound), or NOTHING (in black, no sound), and also showed a running total of earnings. (B) The trial sequence in the spatial orienting task. After a 600-msec "get ready" prompt, a central fixation cross appeared and remained visible throughout the trial. After $500 \mathrm{msec}$, a face cue appeared for $85 \mathrm{msec}$ that was $6.47^{\circ}$ to the left or to the right of fixation. After cue offset, the fixation cross brightened for $15 \mathrm{msec}$. A target then appeared either immediately or after $1,400 \mathrm{msec}$, making a cue-target onset asynchrony (CTOA) of $100 \mathrm{msec}$ or of $1,500 \mathrm{msec}$, respectively. The target appeared for $200 \mathrm{msec}$ to the left or to the right $\left(6.47^{\circ}\right)$ of fixation, just below the previously cued or uncued location. Participants reported target location (left/right) as quickly and as accurately as possible.

ties (EV, valence, or predictability), and congruency (cued, uncued) were conducted. Facilitation and IOR were calculated as an uncued RT minus a cued RT. Alpha levels were set at .05.

\section{RESULTS}

\section{Learning Phase}

Thirty-eight participants demonstrated learning of at least one gain pair (mean learning $=87 \%, S D=9 \%$ ); a partially different subset of 30 participants showed learning for at least one loss pair (mean learning $=80 \%, S D=$ $8 \%$ ); and 28 participants demonstrated learning for at least
Table 1

Results of an ANOVA of Mean Response Time in an Orienting Task for Gain Learners

\begin{tabular}{lcccc}
\hline \multicolumn{1}{c}{ Factors } & $d f$ & $F$ & $p$ & $\begin{array}{c}\text { Effect } \\
\text { Size }\left(\eta_{\mathrm{p}}^{2}\right)\end{array}$ \\
\hline CTOA (100 msec, 1,500 msec) & 1,37 & 39.685 & $<.001$ & .517 \\
Congruency (cued, uncued) & 1,37 & 10.706 & $<.002$ & .224 \\
CTOA $\times$ congruency & 1,37 & 18.125 & $<.001$ & .330 \\
EV $(0, .2 v, .8 v)$ & 2,74 & 4.216 & $<.02$ & .102 \\
EV $\times$ CTOA & & $<1$ & & \\
EV $\times$ congruency & 2,74 & 2.573 & $<.09$ & .065 \\
EV $\times$ CTOA $\times$ congruency & & $<1$ & & \\
\hline
\end{tabular}

Note-CTOA, cue-target onset asynchrony; EV, expected values.

one pair of each type (mean gain learning $=87 \%, S D=$ $9 \%$; mean loss learning $=80 \%, S D=8 \%$ ). Gain learning was significantly better than loss learning $(p<.05)$, but the magnitude of this effect was small $(7 \%)$.

\section{RT: Reward-Associated Cues}

An ANOVA of mean RTs for conditions using EV+ and EV0 cues using CTOA, congruency, and cue EV showed the expected pattern of significant main effects of CTOA and congruency and a significant interaction of these factors (see Table 1 for statistics). As expected, short CTOAs yielded facilitation, and long CTOAs produced IOR. Of relevance was a significant main effect of cue EV and a marginally significant interaction between cue EV and congruency (Table 1). Although the interaction between cue EV and CTOA (and the triple interaction) was nonsignificant, we plotted the results separately for each CTOA to ease interpretation. Figure 2 shows that cues previously associated with reward slowed the RT for targets at cued locations.

We then reanalyzed the data separately for each congruency condition. The main effect of cue EV was significant only for cued targets $\left[F(2,74)=5.552, p=.006, \eta_{\mathrm{p}}^{2}=\right.$

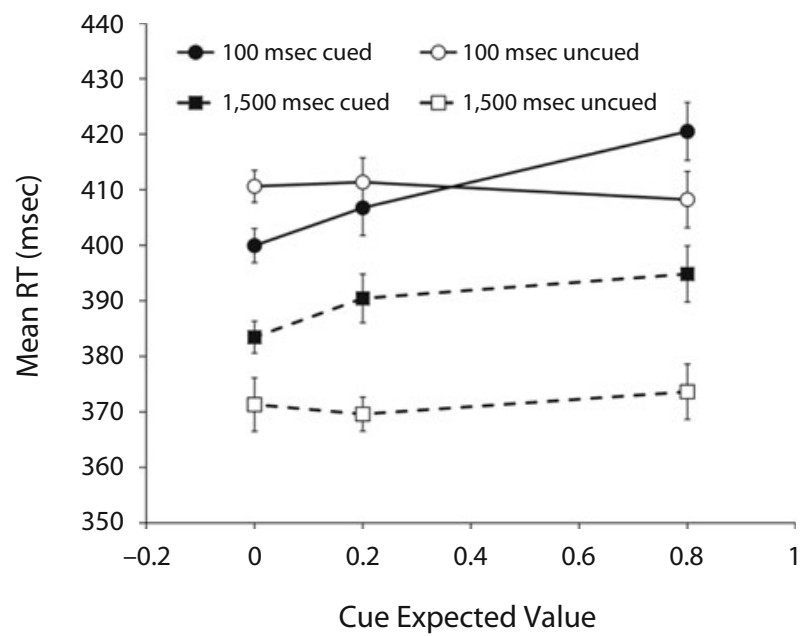

Figure 2. The mean response time (RT) to locate a target in the orienting task, plotted as a function of nominal cue expected value for reward-associated cues. The RT to locate a target presented at either the cued location (closed symbols) or the uncued location (open symbols) is shown for short (100-msec) CTOA (circles, solid lines) and long (1,500-msec) CTOA (squares, dashed lines) conditions. Vertical lines represent a \pm 1 (within-subjects) $S E$. 
.130; uncued, $F<1]$. In neither case did cue EV interact significantly with CTOA $\left(F_{\mathrm{S}}<1\right)$. RTs for cued targets were on average $21 \mathrm{msec}$ slower when preceded by $.8 \mathrm{v}$ cues as compared with EV0 cues $[t(37)=3.38, p<.01]$. Other comparisons were nonsignificant.

When CTOA was short, EV0 cues produced significant facilitation [11 msec; $t(37)=2.53, p<.02$ ], whereas $.8 v$ cues produced an IOR-like effect $[-13 \mathrm{msec} ; t(37)=$ $1.61, p=.11]$. The difference in the size of these effects was significant $(p<.05)$. With a long CTOA, a significant IOR effect was seen for all cue EVs $(p<.005$ in all cases). However, IOR was significantly larger for rewardassociated cues (mean of $.8 v$ and $.2 v=21 \mathrm{msec}$ ) than for EV0 cues $[M=12 \mathrm{msec} ; t(37)=2.08, p<.05]$.

\section{RT: Punishment-Associated Cues}

When RTs for conditions using EV- and EV0 cues were analyzed (Figure 3 ), the expected significant main effects of CTOA, congruency, and their interaction were observed (Table 2). In contrast with the significant EV effect

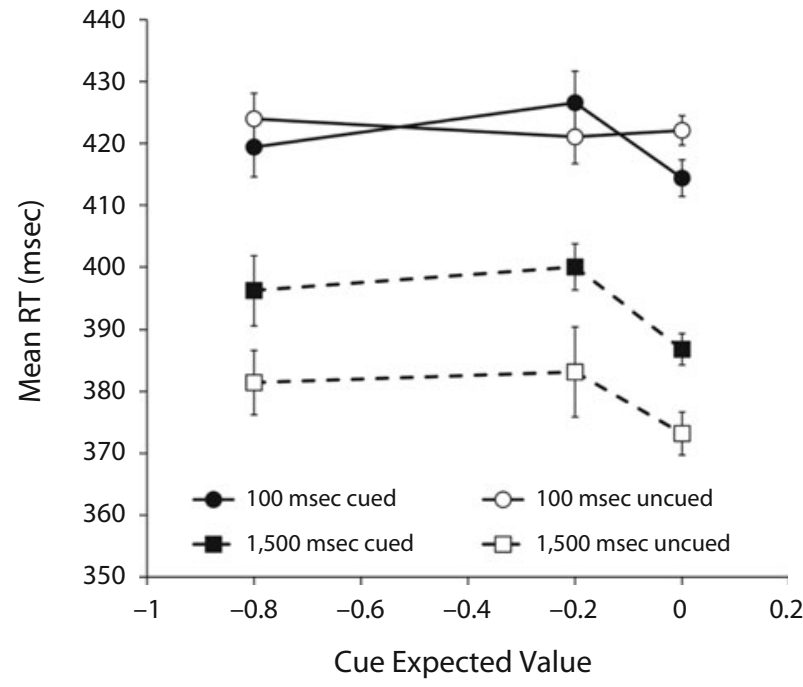

Figure 3. The mean response time (RT) to locate a target in the orienting task, plotted as a function of nominal cue expected value for punishment-associated cues. The RT to locate a target presented at the cued location (closed symbols) or uncued location (open symbols) is shown for short (100-msec) CTOA (circles, solid lines) and long (1,500-msec) CTOA (squares, dashed lines) conditions. Vertical lines represent a \pm 1 (within-subjects) $S E$.

Table 2

Results of an ANOVA of Mean Response Time in an Orienting Task for Loss Learners

\begin{tabular}{lcrlc}
\hline \multicolumn{1}{c}{ Factors } & $d f$ & \multicolumn{1}{c}{$F$} & \multicolumn{1}{c}{$p$} & $\begin{array}{c}\text { Effect } \\
\text { Size }\left(\eta_{\mathrm{p}}^{2}\right)\end{array}$ \\
\hline CTOA $(100 \mathrm{msec}, 1,500 \mathrm{msec})$ & 1,29 & 36.332 & $<.001$ & .556 \\
Congruency $($ cued, uncued) & 1,29 & 6.275 & $<.02$ & .178 \\
CTOA $\times$ congruency & 1,29 & 12.550 & $<.001$ & .302 \\
EV $(0, .2 v, .8 v)$ & 2,58 & 2.970 & $<.06$ & .093 \\
EV $\times$ CTOA & & $<1$ & & \\
EV $\times$ congruency & $<1$ & & \\
EV $\times$ CTOA $\times$ congruency & & $<1$ & & \\
\hline
\end{tabular}

Note-CTOA, cue-target onset asynchrony; EV, expected values.

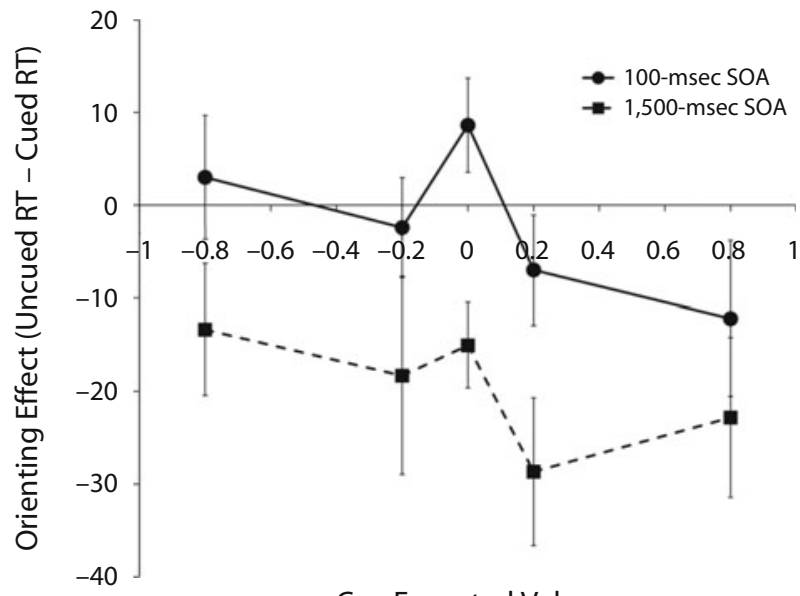

Cue Expected Value

Figure 4. The mean orienting effect (uncued response time [RT] - cued RT), plotted as a function of nominal cue expected value for a subset of 28 participants who learned both gain and loss pairs to criterion. Circles represent data obtained in the short (100-msec) CTOA; squares represent data obtained in the long (1,500-msec) CTOA condition. Vertical lines represent a \pm 1 (within-subjects) $S E$.

for reward-associated cues, the EV effect for punishmentassociated cues was only marginally significant, and its interactions with congruency and CTOA were nonsignificant. For short and long CTOA conditions, it is obvious that the modest (7-msec) slowing effect of punishmentassociated cues on RT was unaffected by CTOA or congruency. Neither the magnitude of facilitation nor IOR varied with cue $\mathrm{EV}$ (both $F_{\mathrm{S}}<1$ ).

\section{Comparing Reward and Punishment}

Lastly, we analyzed data from the subset of participants who passed the learning criterion for at least one gain and one loss pair, thus allowing a direct comparison of reward- versus punishment-associated cues. For this analysis, unlike for the previous ones, we analyzed orienting effects (facilitation, IOR) and excluded data from the EV0 condition so that the effects of EV valence (gain, loss) and probability (high, low) could be treated as separate, within-subjects variables. Interestingly, the main effect of cue valence $\left[F(1,27)=4.642, p<.05, \eta_{\mathrm{p}}^{2}=.147\right]$ was significant. Figure 4 shows that orienting effects were more negative with $\mathrm{EV}+$ cues $(M=-17.7 \mathrm{msec})$ than with EV - cues (mean $=-7.7 \mathrm{msec}$ ). Furthermore, EV+ cues (but not EV - cues) produced more negative orienting effects than EV0 cues $[M=-3.2 \mathrm{msec} ; t(27)=2.90$, $p<.01]$. As expected, there was a significant effect of $\operatorname{CTOA}\left[F(1,27)=7.661, p<.02, \eta_{\mathrm{p}}^{2}=.221\right]$, with orienting effects being more negative with long CTOA than with short CTOA. All other main and interaction effects were nonsignificant (all $F \mathrm{~s}<1$ ).

\section{DISCUSSION}

After learning to associate different faces with different outcomes, participants engaged in a spatial orienting 
task in which these faces served as irrelevant cues. Three important and somewhat surprising results were found. First, RTs obtained in the orienting task were significantly slowed (by $21 \mathrm{msec}$ ) by the brief, prior presentation of irrelevant EV + cues as compared with equally familiar EV0 cues. The corresponding effect for EV - cues was nonsignificant $(7 \mathrm{msec})$ and did not vary by condition. Second, value effects of $\mathrm{EV}+$ cues were evident only when the target appeared at the cued, not uncued, location. Third, value effects were independent of the cue-target interval. The combined effect of these outcomes was that when an EV + cue was presented, slowing instead of facilitation was observed with short CTOAs; with long CTOAs, IOR was significantly enhanced. EV - cues had a weak slowing effect, eliminating facilitation with short CTOAs and leaving IOR unaffected when CTOA was long. These findings provide clear evidence that rapid visual orienting mechanisms are sensitive to value prediction codes that are associated with irrelevant stimuli and raise several interesting questions.

First, why were responses to targets slowed, not speeded, after the presentation of EV + cues, and, second, why were these value prediction effects localized to cued locations only? According to widely accepted notions that the allocation of attention is spatially limited, attentional bias toward reward-associated cues should have resulted in faster (not slower) responses to EV+ cued locations and slower responses to EV+ uncued locations. If an attentional "spotlight" were drawn to the cued location, it should consequently also be slow to arrive at an uncued location. Neither effect was found in the present study, indicating that a spatially limited attention mechanism cannot explain our results. Thus, in contrast with dot-probe studies of voluntary attention using motivationally salient stimuli (e.g., Bradley et al., 2008), our results cannot be characterized by attentional bias. Nevertheless, our finding that reward-associated cues slow target localization clearly indicates that value prediction codes are able to exert a local effect on visual processing. The failure of previous reflexive orienting studies (Lange et al., 2008; Stoyanova et al., 2007) to find effects of emotional cues (e.g., emotional faces) suggests that the stimuli used were not rewarding and that emotional and motivational stimuli activate different neural mechanisms. Our finding reward-associated slowing for targets appearing at cued locations, with no effect at uncued locations, precludes any possibility that these value prediction effects result from central processes related to response selection, such as partial activation of inappropriate choice responses. If this had occurred, then slowing by EV+ cues versus EV0 cues should have been found for both cued and uncued targets. Finally, eye movements also cannot explain the results. Although unlikely (stimuli appeared close to fixation; their location and target onset time were unpredictable), eye movements may have occurred for long CTOAs. However, CTOA and EV did not interact, and EV effects at uncued locations were absent, arguing against an eyemovement-based account.

To account for the value prediction effects of irrelevant cues on visual orienting observed in the present study, we turn to adaptive models of IOR (Dukewich, 2009; Sereno et al., 2010). Such models assume that when the cue and the target appear at the same location, response to the target is reduced by an adaptive gain control mechanism (see Figure 5). Activated by the cue's appearance, gain control is maximally effective shortly after cue onset and diminishes in effect slowly over a lengthy period of time. It acts locally, so it has no effect at uncued locations. Thus, for cued targets and short CTOAs, the gain controller maximally suppresses response. The diminished target response is nevertheless added to the remaining response to the cue, making the summed signal arising at the cued location at, say, $100 \mathrm{msec}$ after target onset greater than that generated at the uncued location in response to the target alone. Thus, overt facilitation effects are seen. When CTOA is long, the target appears after the response to the cue has largely faded. However, the gain controller remains effective, reducing the response to cued targets relative to that of uncued targets (Prime \& Ward, 2004), and IOR effects are observed.

This elegant, physiologically plausible explanation of CTOA effects on visual orienting can neatly explain the present results if we postulate that reward association deepens the initial level of gain control (with all other processes unaffected; Figure 5). This suppresses the response to a cued target with short CTOA after a rewardassociated cue more than it does after an EV0 cue. The summed response in the former case $100 \mathrm{msec}$ after target onset would be below that produced at the same time by an uncued target, producing IOR (as seen in the present study). The slow recovery of the gain function explains why IOR effects at the long CTOA are larger with rewardassociated cues than with EV0 cues. Supporting the possibility of a reward-sensitive adaptive gain control mechanism are findings of repetition-suppression-like effects in the lateral intraparietal area (Lehky \& Sereno, 2007), in a dorsal stream region associated with reward prediction

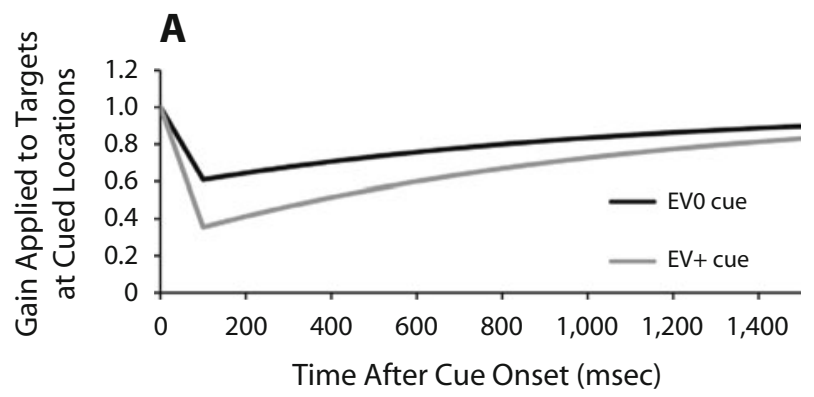

Figure 5. An adaptive gain control model of responses to cues and targets. An initial neural response to all inputs is assumed to rapidly rise and then exponentially decay. This response to cues activates an adaptive gain controller that suppresses subsequent inputs (targets) appearing at the same location. (A) Hypothetical gain control functions for cues with no outcome association (black line) or reward associations (gray line). Gain is lowest $100 \mathrm{msec}$ after the cue onset and slowly improves to reach a value of 1.0 about 3,000 msec later. Reward association is proposed to exacerbate gain control. Thus, responses to cued targets are reduced, relative to those to uncued targets, and this differential is greater with reward-associated cues. 

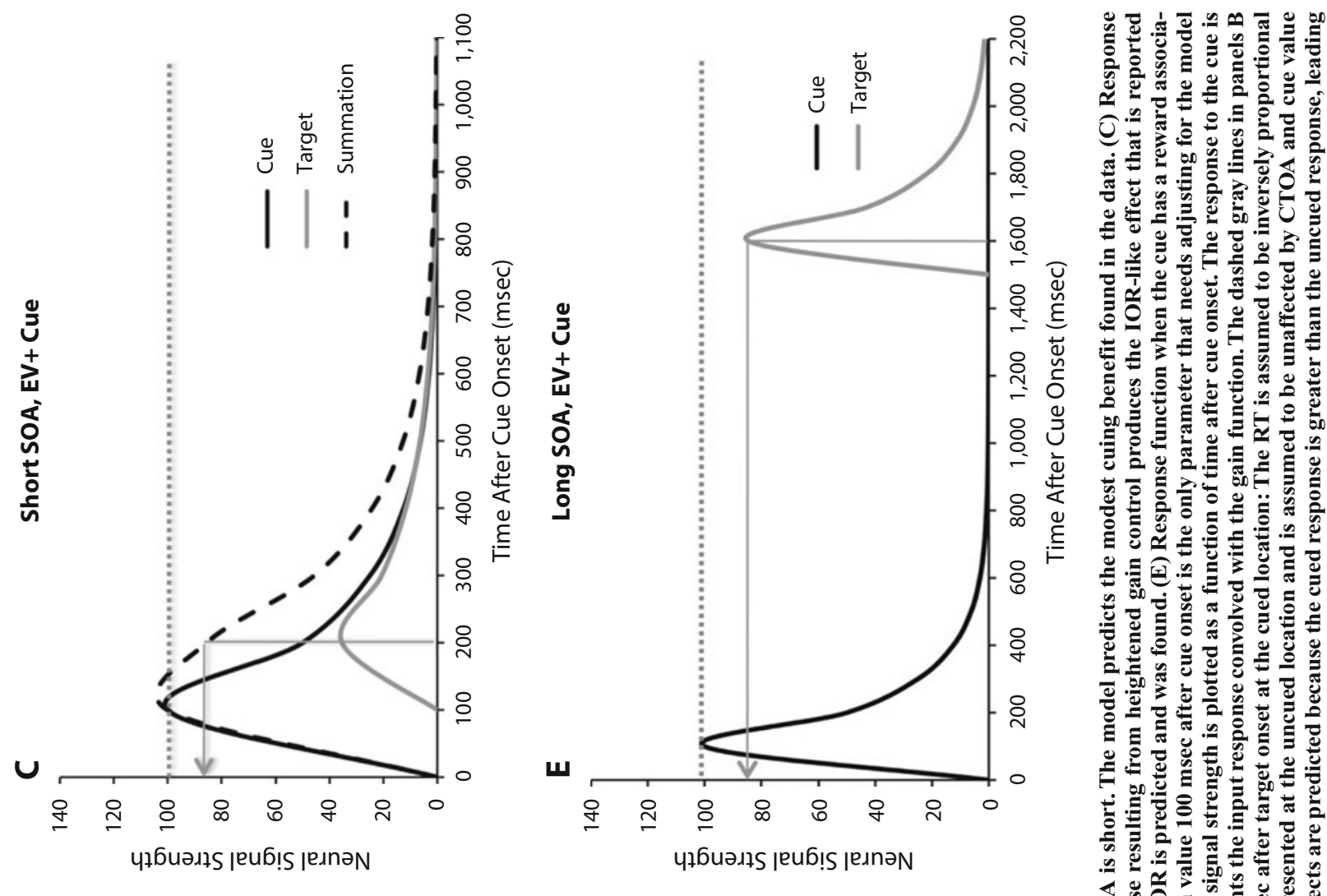

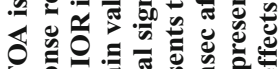

(1)
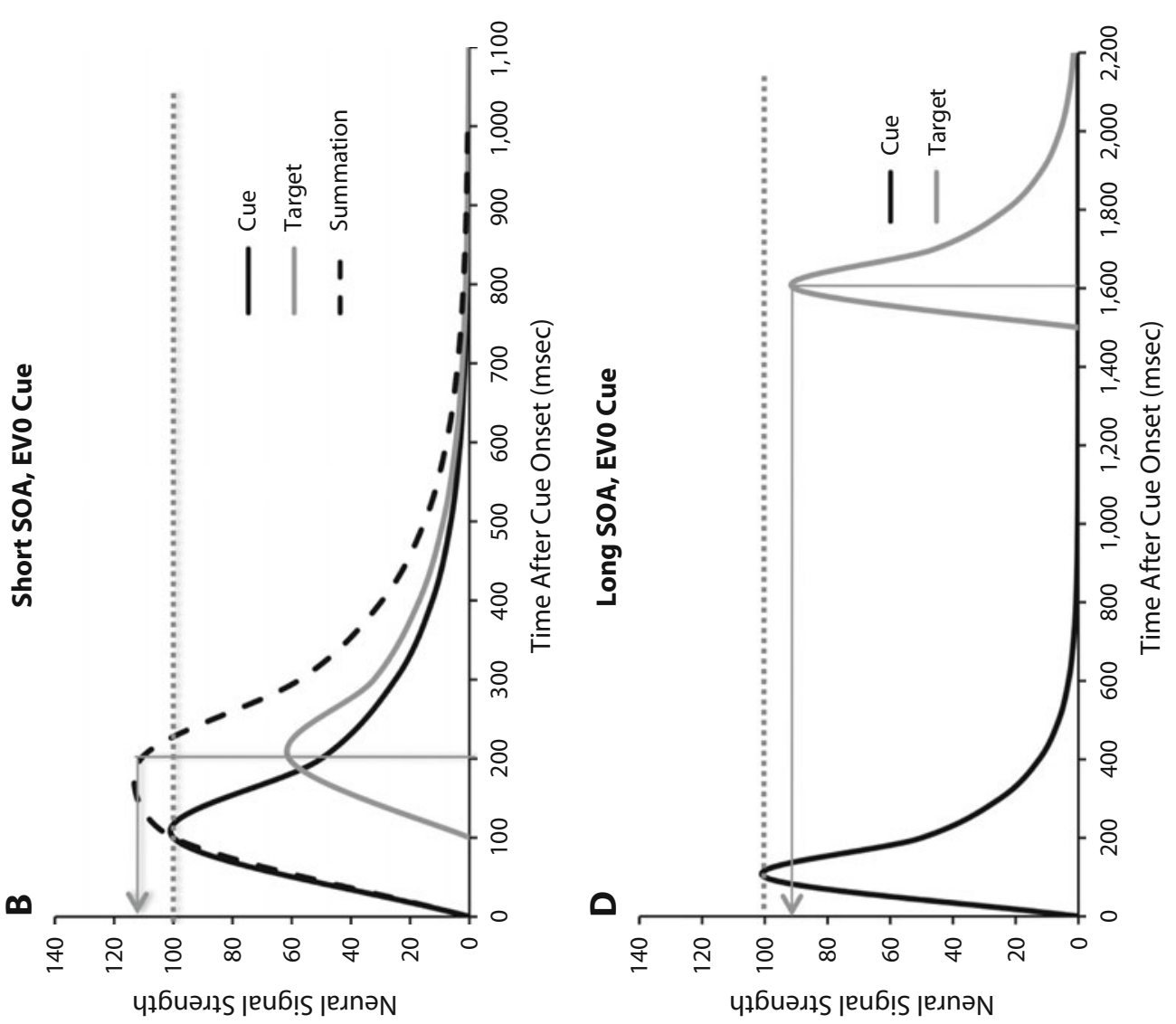

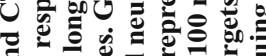

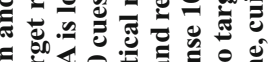
పั

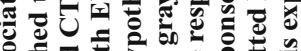

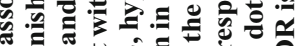
药

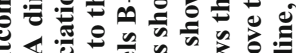

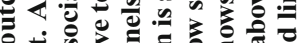

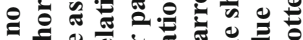

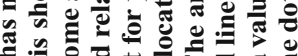

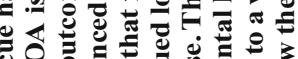

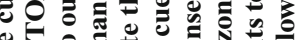
记等 늘

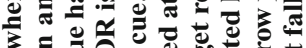
完

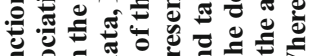

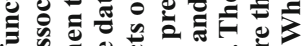

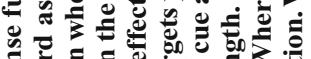

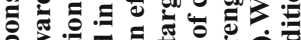

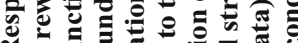

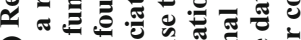

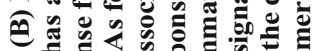
के

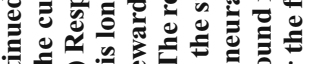

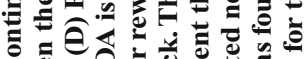
记

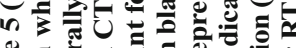

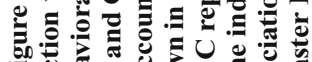
Whing 
(Bendiksby \& Platt, 2006), and in the superior colliculus (Dorris, Klein, Everling, \& Munoz, 2002), an area thought to play a central role in the expression of IOR. Although somewhat speculative, local, adaptive models of CTOA effect on visual orienting provide a plausible account of the value prediction effects that are presently reported.

Finally, we consider our finding that reward-associated cues were more effective at slowing responses than were punishment-associated cues. This finding is consistent with that of a previous study that used a similar conditioning paradigm and stimuli to show that reward-associated but not punishment-associated stimuli "survive" the attentional blink, indicating that the former receive facilitated processing (Raymond \& O'Brien, 2009). A differential effect of valence is also supported by neurophysiological evidence that different neural mechanisms mediate reward versus punishment value learning (Kahn et al., 2002). However, a simpler explanation for the effect of valence seen in the present study is that reward learning was modestly (only 7\%) better than punishment learning. But, when we reanalyzed the data, excluding poor loss learners and good gain learners (equating learning to $83 \%$ ), we still found that EV+ cues significantly slowed responding to cued targets [16 msec; $t(17)=3.43, p<.01]$, as compared with the EV0 trials; the corresponding effect for EV - cues remained small and marginally significant $[8 \mathrm{msec} ; t(17)=1.97, p=.07]$. Consistent with the latter finding, Armony and Dolan (2002) also reported a null effect of aversively conditioned versus neutral cues in a similar, single-cue experiment using a short CTOA.

By manipulating the motivational value of taskirrelevant spatial cues, we demonstrated a clear value prediction effect of irrelevant stimuli on rapid visual orienting. This effect is best explained by value prediction codes rapidly modulating low-level sensory responses and is inconsistent with notions of attentional bias. Our results show that dynamic visual scene processing may be markedly influenced by the presence of previously rewarding objects and suggest that neural codes for prior experience of reward modulate by low-level sensory areas, thus contributing to visual selection.

\section{AUTHOR NOTE}

H.J.V.R. and J.L.O. were supported by studentships from the ESRC (U.K.) and an Overseas Research Students Awards Scheme, respectively. Correspondence concerning this article should be addressed to J. E. Raymond, School of Psychology, Bangor University, Brigantia Building, Penrallt Road, Bangor, Gwynedd LL57 2AS, Wales (e-mail: j.raymond@bangor.ac.uk).

\section{REFERENCES}

Armony, J. L., \& Dolan, R. J. (2002). Modulation of spatial attention by fear-conditioned stimuli: An event-related fMRI study. Neuropsychologia, 40, 817-826.

Bendiksby, M. S., \& Platt, M. L. (2006). Neural correlates of reward and attention in macaque area LIP. Neuropsychologia, 44, 2411-2420.

Bradley, B. P., Field, M., Healy, H., \& Mogg, K. (2008). Do the af- fective properties of smoking-related cues influence attentional and approach biases in cigarette smokers? Journal of Psychopharmacology, 22, 737-745.

BRUCE, G., \& Jones, B. T. (2006). Methods, measures, and findings of attentional bias in substance use, abuse, and dependence. In R. W. Wiers \& A. W. Stacy (Eds.), Handbook of implicit cognition and addiction (pp. 135-149). Thousand Oaks, CA: Sage.

Dorris, M. C., Klein, R. M., Everling, S., \& Munoz, D. P. (2002). Contribution of the primate superior colliculus to inhibition of return. Journal of Cognitive Neuroscience, 14, 1256-1263.

Dukewich, K. R. (2009). Reconceptualizing inhibition of return as habituation of the orienting response. Psychonomic Bulletin \& Review, 16, 238-251

Engelmann, J. B., \& Pessoa, L. (2007). Motivation sharpens exogenous spatial attention. Emotion, 7, 668-674.

Fox, E., Russo, R., \& Dutton, K. (2002). Attentional bias for threat: Evidence for delayed disengagement from emotional faces. Cognition \& Emotion, 16, 355-379.

Kahn, I., Yeshurun, Y., Rotshtein, P., Fried, I., Ben-Bashat, D., \& HENDLER, T. (2002). The role of the amygdala in signaling prospective outcome of choice. Neuron, 33, 983-994.

Kiss, M., Driver, J., \& Eimer, M. (2009). Reward priority of visual target singletons modulates event-related potential signatures of attentional selection. Psychological Science, 20, 245-251.

Lange, W.-G., Heuer, K., Reinecke, A., Becker, E. S., \& Rinck, M. (2008). Inhibition of return is unimpressed by emotional cues. Cognition \& Emotion, 22, 1433-1456.

LEHKY, S. R., \& SERENO, A. B. (2007). Comparison of shape encoding in primate dorsal and ventral visual pathways. Journal of Neurophysiology, 97, 307-319.

O’DoherTy, J. P. (2004). Reward representations and reward-related learning in the human brain: Insights from neuroimaging. Current Opinion in Neurobiology, 14, 769-776.

Posner, M. I., \& CoHEN, Y. A. (1984). Components of visual orienting. In H. Bouma \& D. G. Bouwhuis (Eds.), Attention and performance X: Control of language processes (pp. 531-554). Hillsdale, NJ: Erlbaum.

Posner, M. I., Rafal, R. D., Choate, L. S., \& Vaughan, J. (1985). Inhibition of return: Neural basis and function. Cognitive Neuropsychology, 2, 211-228

PRIME, D. J., \& WARD, L. M. (2004). Inhibition of return from stimulus to response. Psychological Science, 15, 272-276.

RAYMOND, J. E., \& O'BRIEN, J. L. (2009). Selective visual attention and motivation: The consequences of value learning in an attentional blink task. Psychological Science, 20, 981-988.

Robinson, T. E., \& BerRidge, K. C. (1993). The neural basis of drug craving: An incentive-sensitization theory of addiction. Brain Research Reviews, 18, 247-291.

Schneider, W., Eschman, A., \& Zuccolotto, A. (2002). E-Prime user's guide. Pittsburgh: Psychological Software Tools.

Sereno, A. B., Lehky, S. R., Patel, S. S., \& Peng, X. (2010). A neurophysiological correlate and model of reflexive spatial attention. In N. Srinivasan, B. R. Kar, \& J. Pandey (Eds.), Advances in cognitive science (Vol. 2, pp. 104-131). Thousand Oaks, CA: Sage.

Small, D. M., Gitelman, D., Simmons, K., Bloise, S. M., Parrish, T., \& Mesulam, M.-M. (2005). Monetary incentives enhance processing in brain regions mediating top-down control of attention. Cerebral Cortex, 15, 1855-1865.

Stolarova, M., Keil, A., \& Moratti, S. (2006). Modulation of the $\mathrm{C} 1$ visual event-related component by conditioned stimuli: Evidence for sensory plasticity in early affective perception. Cerebral Cortex, 16, 876-887.

Stoyanova, R. S., Pratt, J., \& Anderson, A. K. (2007). Inhibition of return to social signals of fear. Emotion, 7, 49-56.

(Manuscript received August 13, 2009; revision accepted for publication February 8, 2010.) 